\title{
hot off the press
}

\section{SIRT3 deacetylase: the Jekyll and Hyde sirtuin}

\author{
Dafne M. Silberman \& Raul Mostoslavsky
}

$\mathrm{P}$ ost-translational modifications have crucial roles in regulating the functions of many eukaryotic proteins. Among them, lysine acetylation has been traditionally studied in the context of nuclear histone modifications, and was one of the first to be described as part of the 'histone code' hypothesis (Kim et al, 2006). More recently, work from several groups has demonstrated that lysine acetylation also modulates the activity of several non-histone proteins. In this context, this modification seems particularly abundant on mitochondrial proteins (Schwer et al, 2009). However, the way in which acetylation influences enzyme function and metabolic reprogramming in pathological states remains unknown. In this issue of EMBO reports, Sack and colleagues shed new light on the role of mitochondrial SIRT3 deacetylase during paracetamol-induced toxicity, describing the mitochondrial protein aldehyde dehydrogenase $2(\mathrm{ALDH} 2)$ as a new target of SIRT3, and a protective role for protein acetylation in this context (Lu et al, 2011).

\section{...these results raise the tantalizing possibility that —at least in the context of [paracetamol] toxicity-the less SIRT3 the better}

The sirtuin family of $\mathrm{NAD}^{+}$-dependent deacetylases comprises seven mammalian homologues (SIRT1-SIRT7) that have diverse functions and cellular localizations (Finkel et al, 2009). Among them, mitochondrial SIRT3 is the main deacetylase involved in the modulation of mitochondrial metabolic and oxidative-stress regulatory pathways (Schwer et al, 2009). SIRT3 seems to mediate protection against oxidative damage under caloric restriction (Someya et al, 2010), as well as promoting enhanced protection against redox and nutrient-excess stress (Zhong \& Mostoslavsky, 2011).

Acetaminophen (APAP)—commonly known as paracetamol-is a widely used analgesic and anti-pyretic drug that is safe at therapeutic-dose levels. However, APAP overdose has been linked to liver injury in both humans and mice (Jaeschke \& Bajt, 2006), with a high mortality rate due to acute liver failure. Remarkably, this hepatotoxic effect seems to be enhanced by fasting (Whitcomb \& Block, 1994), a phenomenon that was poorly understood. In the initial phases of cell injury, a product of APAP oxidation-the highly reactive metabolite $\mathrm{N}$-acetyl-p-benzoquinoneimine (NAPQI) binds to protein cysteine and lysine residues (Zhou et al, 1996), eventually depleting hepatic glutathione and leading to the concomitant hepatotoxicity. Although there has been extensive research, the underlying molecular mechanisms of liver injury have not been fully elucidated.

In this new study, Lu and colleagues aimed to decipher the way in which fasting or caloric-restriction exacerbate the redox-stress-dependent toxicity of APAP (Lu et al, 2011). Given the known increase in SIRT3 activity on nutrient deprivation, they proposed that, if protein acetylation inhibits NAPQI binding, SIRT3mediated deacetylation might aggravate acetaminophen-induced liver injury (AILI).

First, they tested whether lack of SIRT3 protects against AILI, by analysing susceptibility to liver injury in $\mathrm{SIRT}^{+/+}$and $\mathrm{SIRT3}^{-1-}$ mice treated with a single toxic dose of APAP under fed and fasted conditions. Strikingly, they found that fasted $\mathrm{SIRT}^{-/-}$mice showed less hepatotoxicity than the SIRT3-competent mice. By using two-dimensional gel and immunoblot analyses, they then compared hepatic mitochondrial-protein acetylation profiles between fasted $\mathrm{SIRT}^{-/-}$and SIRT3 ${ }^{+/+}$mice. In these experiments they identified, among several candidates, ALDH2-a known target of NAPQI, binding to which is known to reduce ALDH2 activity (Landin et al, 1996). This dehydrogenase oxidizes and detoxifies aldehydes_including lipid peroxidation products such as trans-4-hydroxy-2-nonenal (4-HNE; Doorn et al, 2006)—and thus buffers these highly reactive metabolites.

\section{...SIRT3 might act as a double- edged sword [raising] a word of caution regarding therapeutic strategies aimed at potentiating SIRT3 activity}

$\mathrm{Lu}$ and colleagues then focused on $\mathrm{ALDH} 2$. In a series of elegant studies, they demonstrated that ALDH2 is a direct target of SIRT3, and deacetylation of ALDH2 modifies NAPQI binding. Liver mitochondria from SIRT3-deficient mice had increased $\mathrm{ALDH} 2$ acetylation, indicating a direct interaction between SIRT3 and ALDH2. $\mathrm{ALDH} 2$ was then shown to be a direct target of SIRT3 by using in vitro deacetylation assays. Despite these differences, basal $\mathrm{ALDH} 2$ activity remained the same in both genotypes; enzymatic activity was therefore evaluated in response to APAP treatment in fasted mice. Remarkably, SIRT3-deficient mitochondria exhibited approximately $40 \%$ higher levels of ALDH2 activity after APAP administration and, consequently, significantly lower levels of 4-HNE adducts were detected, in comparison to SIRT3 $3^{+/+}$mice. SIRT3 is a known protective factor against oxidative stress; however, these results raise the tantalizing possibility that-at least in the context of APAP toxicity-the less SIRT3 the better.

Logically, the next step was to show that the protective effect of SIRT3 deficiency is directly dependent on sustained ALDH2 activity. A marked increased in liver injury in the SIRT3-deficient animals was observed 
after knockdown of ALDH2 by using a lentiviral short-hairpin RNA approach, supporting their argument. To gain further molecular insight, the authors followed previous observations indicating that binding of NAPQI to ALDH2 diminishes ALDH2 activity (Landin et al, 1996). They hypothesized that SIRT3 might deacetylate ALDH2, in turn increasing its binding to NAPQI and leading to the concomitant inactivation of the protein. Indeed, through elegant SIRT3 gain- and loss-of-function experiments, they demonstrated that SIRT3-dependent deacetylation of ALDH2 enhances binding of the enzyme to NAPQI, whereas SIRT3 inactivation decreases NAPQI binding to ALDH2.

The Sack group went one step further and used mass spectrometry to identify ALDH2 Lys 377 as the residue deacetylated by SIRT3. They showed that acetylation of Lys 377 is increased in SIRT3-deficient mice, and a mutant ALDH2 with an acetylmimicking mutation (K377Q) exhibited significantly less binding to NAPQI, giving a detailed molecular explanation for the protective effect observed in the absence of this sirtuin.

These findings demonstrate that SIRT3mediated deacetylation of mitochondrial proteins modulates susceptibility to AILI. Furthermore, the identification of ALDH2 as the substrate for SIRT3 deacetylation in this process provides a molecular framework in which to understand the apparent paradox of enhanced APAP toxicity under conditions of fasting or caloric restriction. Fasting induces SIRT3-mediated deacetylation of ALDH2, leading to increased NAPQI binding, which in turn reduces ALDH2 activity. This causes an accumulation of highly reactive adducts, probably contributing to the exacerbated hepatotoxicity observed after APAP treatment under nutrient restriction (Fig 1).

The toxic effects of AILI have been traditionally addressed by using antioxidant therapies based on NAPQI binding to cysteine residues. Surprisingly, the functional outcome of NAPQI binding to lysine residues has not been explored so far, although it was described almost 15 years ago (Zhou et al, 1996). The Sack

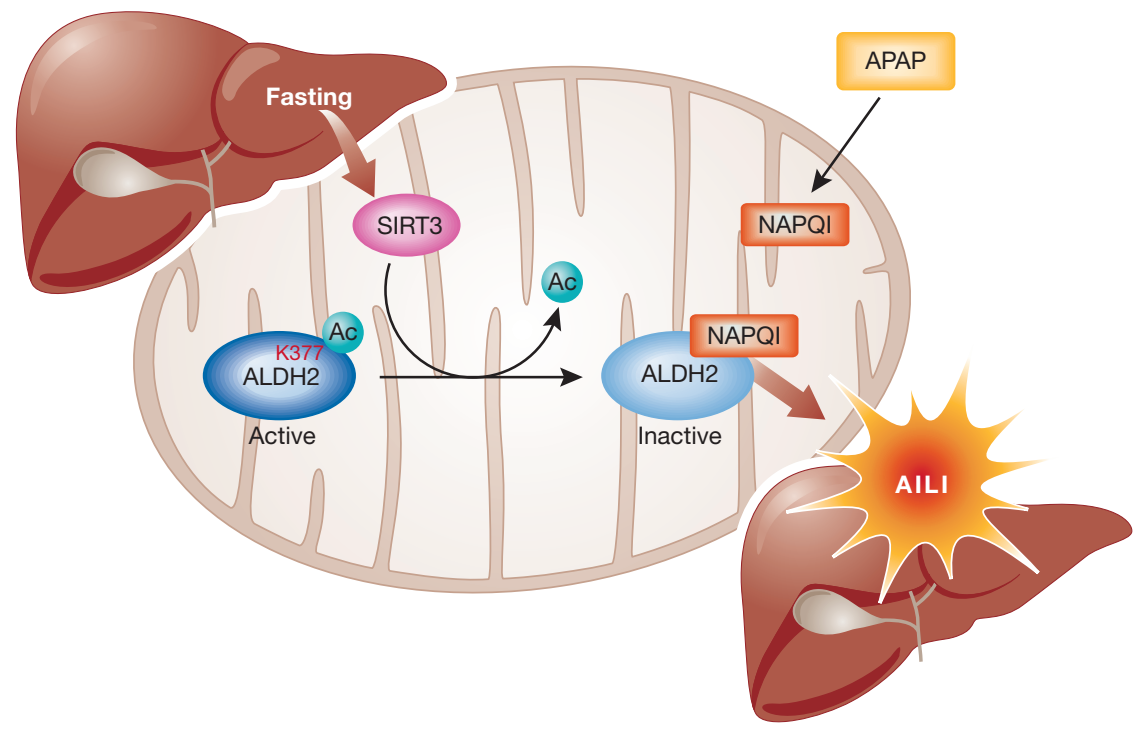

Fig 1 | SIRT3-mediated exacerbation of acetaminophen-induced liver injury. SIRT3 deacetylates Lys 377 of ALDH2, making it available for NAPQI binding, which de-activates it. The concomitant reduction in the aldehyde-detoxifying activity of ALDH2 aggravates liver injury. AILI, acetaminophen-induced liver injury; ALDH2, aldehyde dehydrogenase 2; NAPQI, N-acetyl-p-benzoquinoneimine.

laboratory approached this issue, providing clear, supportive data for an interesting and provocative hypothesis: although it is widely accepted that SIRT3 has protective, anti-oxidant effects, ALDH2 deacetylation by SIRT3 exacerbates APAP-induced hepatotoxicity. This indicates that SIRT3 might act as a double-edged sword, and raises a word of caution regarding therapeutic strategies aimed at potentiating SIRT3 activity. Although this study provides support for this paradoxical effect, some questions remain. First, is $\mathrm{ALDH} 2$ the only SIRT3 substrate involved in this phenotype? The authors show that several other proteins were identified in their study, but their roles remain to be explored. Second, what is the physiological role of SIRT3-mediated ALDH2 deacetylation? Does this modification alter ALDH2 activity under conditions of nutrient stress? If so, how? Third, how general is this phenomenon? Does protein deacetylation modulate the binding of other toxic metabolites to proteins in detoxifying organs, such as the liver? Although answers to these questions await future investigation, one thing is certain: we need to exercise caution when evaluating the therapeutic potential of sirtuin modulators.

\section{REFERENCES}

Doorn JA et al (2006) Chem Res Toxicol 19: 102-110 Finkel T et al (2009) Nature 460: 587-591

Jaeschke H, Bajt ML (2006) Toxicol Sci 89: 31-41 Kim SC et al (2006) Mol Cell 23: 607-618

Landin JS et al (1996) Toxicol Appl Pharmacol 141: 299-307

Lu Z et al (2011) EMBO Rep12: 840-846

Schwer B et al (2009) Aging Cell 5: 604-606

Someya S et al (2010) Cell 143: 802-812

Whitcomb DC, Block GD (1994) JAMA 272: 1845-1850

Zhong L, Mostoslavsky R (2011) Cell Metab 13: 621-626

Zhou L et al (1996) Chem Res Toxicol 9: 1176-1182

Dafne M. Silberman and Raul Mostoslavsky are at the Massachusetts General Hospital Cancer Center, Harvard Medical School, Boston, USA. Dafne M. Silberman is also at the Department of Human Biochemistry, Medical School/CEFyBO-UBA/CONICET, Buenos Aires, Argentina.

E-mail: rmostoslavsky@mgh.harvard.edu

Published online 8 July 2011

EMBO reports (2011) 12, 746-747.

doi:10.1038/embor.2011.147 